\title{
Laser systems stabilized to cryogenic silicon cavities for precision measurements
}

\author{
Konstantin Kudeyarov ${ }^{1,2, *}$, Nikita Zhadnov ${ }^{1,2}$, Denis Kryuchkov ${ }^{1,2}$, Gulnara Vishnyakova ${ }^{1}$, \\ Ksenia Khabarova ${ }^{1,2}$, and Nikolay Kolachevsky ${ }^{1,2}$ \\ ${ }^{1}$ Lebedev Physical Institute, 1119991 Moscow, Russia \\ ${ }^{2}$ Russian Quantum Center, 121205 Moscow, Russia
}

\begin{abstract}
We consider laser systems stabilized to external Fabry-Perot silicon cavities operated at cryogenic temperatures. In order to characterize frequency stability two identical systems were created. Fractional frequency instability of individual system reached $6 \times 10^{-15}$ at $1 \mathrm{~s}$. Different sources of noises were studied, and the dominant one now is the fluctuations of residual amplitude modulation.
\end{abstract}

Laser sources with ultranarrow (less than $1 \mathrm{~Hz}$ ) spectral linewidths are of great importance in precision measurement science. Its applications include gravitational waves detection [1], search for dark matter [2] and drift of fundamental constants [3], dissemination of ultrastable time and frequency signals [4]. Ultrastable lasers are the vital parts of state-of-art frequency standards - optical clocks, whose instability and systematic uncertainty have reached values below $10^{-18}[5,6]$. Due to outstanding performance optical clock can be used as very precise sensor, for instance, to create a map of Earth gravitational potential [7].

To achieve high stability laser frequency is usually locked to transmission peak of highfinesse Fabry-Perot cavity using well-developed Pound-Drever-Hall method. If all technical noises are suppressed, frequency fluctuations are defined by fluctuations of distance between cavity mirrors. The fundamental limit on frequency stability is imposed by thermal noise of cavity parts: spacer, mirrors substrates and coatings. The reduction of thermal noise can be reached by choosing the appropriate material, decreasing the temperature and increasing the length of cavity [8]. The best laser frequency instability achieved up to date is $4 \times 10^{-17}$ [9].

We developed two lasers systems operating at a wavelength of $1542 \mathrm{~nm}$ based on singlecrystal silicon cavities. Cavities are cooled down to $124 \mathrm{~K}$, where coefficient of thermal expansion of silicon is zero, using liquid nitrogen cryostats of original design [10]. The creation of two identical systems allows to learn the individual stability and to determine what limits it. The fractional frequency instability of our lasers (Fig. 1) is averaged down as white phase noise until $0.01 \mathrm{~s}$, experienced flicker floor near $6 \times 10^{-15}$ at averaging times 0.1 $10 \mathrm{~s}$ and then begins to drift. On the way to thermal noise limit at $2 \times 10^{-16}$ we need to overcome both perturbations that change the length of cavity and noise of optoelectronic feedback loop.

\footnotetext{
*Corresponding author: kost1994@yandex.ru
} 


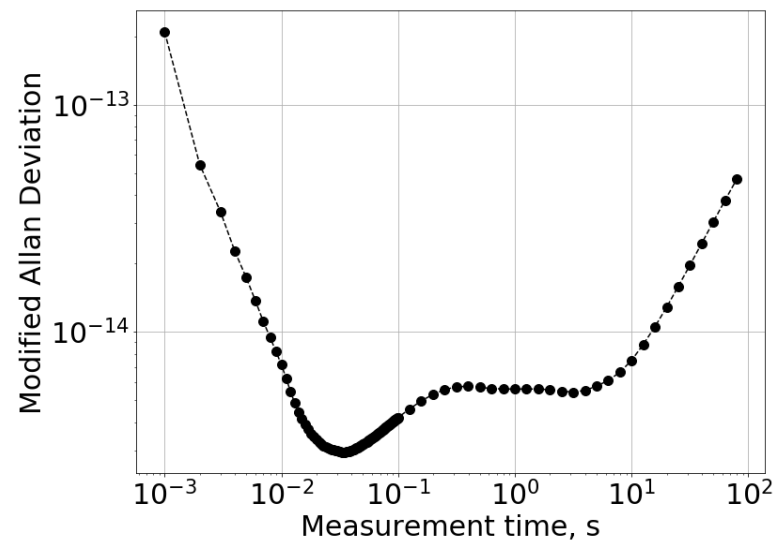

Fig. 1. Fractional frequency instability of laser stabilized to silicon cavity obtained by comparison of two identical systems.

We investigated the influence of temperature fluctuations [11], vibrations, noise of electronics and others. The major instability source in our system is fluctuation of residual amplitude modulation raised by polarization imperfections in electro-optic modulator and parasitic etalons. In order to compensate the influence of residual amplitude modulation we are working on feedback system.

The work is funded and supported by the Russian Science Foundation (grant №19-72-10166).

\section{References}

1. P. Kwee, C. Bogan, K. Danzmann, et al., Opt. Express, 20, 10617(2012)

2. A. A. Geraci, C. Bradley, D. Gao, et al. , Phys. Rev. Lett., 123, 031304 (2019)

3. M. S. Safronova, D. Budker, D. DeMille, et al., Rev. Mod. Phys., 90(2) (2018)

4. S. Droste, T. Udem, R. Holzwarth, T. W. Hänsch, Comptes Rendus Phys., 16, 524 (2015)

5. S. M. Brewer, J.-S. Chen, A. M. Hankin, et al., arXiv:1902.07694

6. E. Oelker, R. B. Hutson, C. J. Kennedy, et al., Nat. Photon. (2019)

7. W. McGrew, X. Zhang, R. Fasano, et al., Nature, 564, 87 (2018)

8. T. Kessler, T. Legero, and U. Sterr, J. Opt. Soc. Am. B, 29, 178 (2012)

9. D. G. Matei, T. Legero, S. Häfner, et al., Phys. Rev. Lett., 118, 263202 (2017)

10. N.O. Zhadnov, A. V. Masalov, V. N. Sorokin, et al., Quantum Electron., 47, 421 (2017)

11. N.O. Zhadnov, G. A. Vishnyakova, K. S. Kudeyarov, et al., Quantum Electron., 49, 424 (2019) 